RESEARCH HIGHLIGHT

\title{
Response-contingent optogenetics to discover the mechanisms of nicotine-cue associations
}

\author{
Victória A. Müller Ewald ${ }^{1,2}$ and Ryan T. LaLumiere ${ }^{1,2,3}$ \\ Neuropsychopharmacology (2019) 44:1995-1996; https://doi.org/10.1038/s41386-019-0469-6
}

Despite the well-known health risks of tobacco use [1], nearly 30 million Americans smoke cigarettes every day (2016 National Survey on Drug Use and Health, September 2017). A major challenge encountered by those who attempt to quit nicotine use is the high likelihood of relapse, often driven by nicotineassociated cues. These cues gain motivational properties of their own when neutral cues (e.g., the sight of a cigarette lighter) are repeatedly paired with nicotine use and can come to elicit craving and precipitate relapse. This raises the possibility that, if the mechanisms for how the brain encodes these cue associations were understood, treatment could directly target such mechanisms as a means of reducing relapse. Clinical evidence indicates that cue-driven nicotine craving involves increased activity in the medial prefrontal cortex (mPFC). Similarly, mPFC activity is a critical component for cue-induced reinstatement in various rodent models of drug seeking, with different functions attributed to dorsal and ventral subdivisions (dmPFC and vmPFC, respectively) [2-5]. In this current issue of Neuropsychopharmacology, Struik et al. report on a series of experiments investigating the role of these subdivisions in encoding nicotine-cue associations during extinction following nicotine self-administration in rats.

During an extinction session in which an instrumental response produces the drug-associated cues, but not the drug itself, the organism updates its knowledge regarding the relationship between the cues and the drug-i.e., that the cues no longer predict the drug. Disruption of this new learning would be expected to increase drug-seeking behavior during the extinction session, as the organism would fail to change its behavior in response to the change in contingencies. This suggests that a specific period of encoding during the extinction training session is responsible for this new learning, necessitating an experimental approach capable of disrupting activity in a temporally limited window corresponding to the encoding, in contrast to pharmacological manipulations that lack such temporal precision.

To this end, Struik et al. used a response-contingent optogenetic approach to control neural activity during discrete time windows in self-administration and extinction training sessions. They trained rats to nose-poke for a nicotine infusion that was paired with 5 -s cues. Following nicotine self-administration, rats underwent extinction tests in which nose-pokes produced the cues but not the nicotine infusion. During extinction, nose-pokes also produced $6 \mathrm{~s}$ of optical inhibition of either the dmPFC or the vmPFC during the cue presentation or $60 \mathrm{~s}$ after the cues (control condition). The results indicate that dmPFC inhibition during the cues, but not $60 \mathrm{~s}$ after the cues, increased nicotine seeking. vmPFC inhibition had no effect on behavior in either condition. As a result, the authors suggest that the dmPFC encodes and updates nicotine-cue associations during extinction as part of its role in guiding goal-directed behavior.

Based on this conclusion, the disrupted encoding would be expected to impair retention of the extinction learning, leading to higher levels of nose-poking in the subsequent extinction session. However, Struik et al. found that dmPFC inhibition did not lead to higher responding in the subsequent extinction session when inhibition was not given. The authors suggest that a different aspect of the extinction of the instrumental learning (e.g., actionoutcome contingency) may be encoded elsewhere and that that aspect was responsible for the between-session extinction. Interestingly, the authors also found that dmPFC inhibition during cue delivery had no effect during self-administration sessions or in the extinction of food seeking. Although the latter finding may seem surprising, it is consistent with our own work using response-contingent optogenetic inhibition during the extinction of cocaine seeking [6]. We found that the vmPFC, rather than the dmPFC, mediated the extinction encoding for cocaine seeking, yet vmPFC inhibition also had no effect on the extinction of food seeking. These findings strongly suggest critical differences between drug-seeking behavior and reward-seeking behavior in general and point to changes in MPFC plasticity and functioning because of chronic drug use.

To further investigate the role of this region in nicotine-cue encoding, the authors conducted in vivo electrophysiological recordings of dmPFC activity during the task and found a significant increase in the variability of dmPFC neuronal firing during extinction trials compared to self-administration trials. The authors suggest that this increase in neuronal firing rate variability is evidence that this region is involved in encoding the change in contingencies surrounding nicotine-associated cues during the extinction session. However, what these changes in firing rate mean in terms of subsequent behavior is unknown and future work should consider how these neurons are encoding the new contingencies for the nicotineassociated cues. Nonetheless, the electrophysiological findings are in agreement with the broader literature that has shown cue-evoked activity in the dmPFC during both reward seeking and extinction conditions in other reward-seeking procedures, including sucrose-seeking [7]. Indeed, work suggests that glutamatergic inputs from the dmPFC modulate

\footnotetext{
${ }^{1}$ Interdisciplinary Neuroscience Program, lowa City, IA 52242, USA; ${ }^{2}$ Department of Psychological and Brain Sciences, University of lowa, lowa City, IA 52242, USA and ${ }^{3}$ lowa Neuroscience Institute, University of lowa, lowa City, IA 52242, USA

Correspondence: Victória A. Müller Ewald (victoriaaugusta-mullerewald@uiowa.edu)
}

Received: 8 July 2019 Accepted: 12 July 2019

Published online: 5 August 2019 
cue-elicited firing in nucleus accumbens core [8], another region that regulates and generally promotes drug-seeking behavior.

Together, these findings suggest the possibility of using a gainof-function approach to enhance encoding and reduce nicotine seeking during extinction. Therefore, the authors used the response-contingent procedure to optogenetically stimulate the dmPFC during extinction trials. Stimulation parameters were chosen based on previous work suggesting that beta oscillations $(20 \mathrm{~Hz})$ in the cortical-basal ganglia network are important for cue utilization in guiding motivated behavior [9]. However, results indicate that this stimulation did not alter the rats' behavior. One potential reason for the lack of effect is that driving neuronal firing patterns is not the same as driving endogenous brain rhythms. Nonetheless, the overall set of findings provides a good basis for future work investigating how MPFC subregions encode nicotinecue associations. Indeed, determining how the brain maintains and updates the contingencies between addictive drugs and cues is a critical step in our understanding of drug addiction. Such findings lay the groundwork for the development of treatments that enhance such updating as a means of reducing the likelihood of relapse.

\section{FUNDING AND DISCLOSURE}

RTL was supported by NIH R01 DA034684. The authors declare no competing interests.

\section{ADDITIONAL INFORMATION}

Publisher's note: Springer Nature remains neutral with regard to jurisdictional claims in published maps and institutional affiliations.

\section{REFERENCES}

1. World Health Organization. WHO report on the global tobacco epidemic: raising taxes on tobacco. Geneva: World Health Organization; 2015. p. 1-103.

2. Bossert JM, et al. Ventral medial prefrontal cortex neuronal ensembles mediate context-induced relapse to heroin. Nat Neurosci. 2011;14:420-2.

3. Lubbers BR, et al. Prefrontal gamma-aminobutyric acid type A receptor insertion controls cue-induced relapse to nicotine seeking. Biol Psychiatry. 2014;76:750-8.

4. LaLumiere RT, Smith KC, Kalivas PW. Neural circuit competition in cocaine-seeking: roles of the infralimbic cortex and nucleus accumbens shell. Eur J Neurosci. 2012;35:614-22.

5. Gourley SL, Taylor JR. Going and stopping: dichotomies in behavioral control by the prefrontal cortex. Nat Neurosci. 2016;19:656-64.

6. Gutman $\mathrm{AL}$, et al. Extinction of cocaine seeking requires a window of infralimbic pyramidal neuron activity after unreinforced lever presses. J Neurosci. 2017:37:6075-86.

7. Moorman DE, Aston-Jones G. Prefrontal neurons encode context-based response execution and inhibition in reward seeking and extinction. Proc Natl Acad Sci USA. 2015;112:9472-7.

8. Ishikawa A, et al. Dorsomedial prefrontal cortex contribution to behavioral and nucleus accumbens neuronal responses to incentive cues. J Neurosci. 2008;28:5088-98.

9. Leventhal DK, et al. Basal ganglia beta oscillations accompany cue utilization. Neuron. 2012;73:523-36. 